\title{
Moving Target Detection Algorithm Research Based on Background Subtraction Method
}

\author{
Songlin Wan ${ }^{1}$, Haodong Liang, Taifang Wang, Zhi Cui
}

\begin{abstract}
Based on Surendra algorithm, an improved adaptive threshold algorithm has been proposed. Simultaneously, a gradient update rate algorithm has also been proposed to better adaptability and robustness in various videos. These two algorithms with real-time and detecting time shorter have been effectively used in moving target detection.
\end{abstract}

Keywords: Background subtraction method, Improved Surendra adaptive threshold algorithm, Gradient update rate algorithm (GUR algorithm)

\section{Introduction}

The video moving objects detection has already been a hot issue in current study around the world. There are some mature algorithms such as Optical Flow Method [1], Frame Difference Method [2], and Background Subtraction Method [3] so far. This article is based on the classic Surendra algorithm [4] [5] of Background Subtraction Method, whatever the threshold in the Surendra algorithm is set to be a fixed value or fixed by some exist algorithm[6] [7] [8] [9], we still could not get a good real-time updating background. So some existed algorithms are summarized, and then a new adaptive threshold algorithm based on Surendra algorithm is proposed. This new adaptive threshold algorithm consumes shorter time, gets better effect than original algorithm, and has practical significances. In addition, the gradient update rate algorithm (GURA) is also raised, due to the discovery that it may bring out a bad background extraction at the beginning of the video, through testing of various kinds of video, the two algorithms both have good robustness and great efficiency.

\footnotetext{
${ }^{1}$ Songlin Wan $(\bowtie)$

School of Optoelectronic Information, University of Electronic Science and Technology of China, Chengdu Sichuan 610054, China

e-mail:w285531068@gmail.com
} 


\section{Moving Target Detection Algorithm}

\subsection{The Overall Algorithm Introduction}

The overall algorithm consists of five parts: the extraction of the background, the background updating, the subtraction of background, the detection of the target, and post treatment.

The background updating and extracting are critical for the complexity of subsequent processing in above algorithm which is shown in Fig. 1:

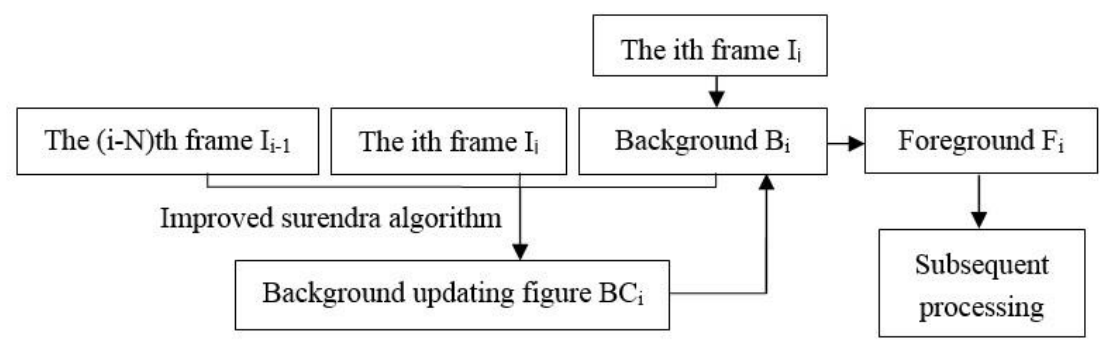

Fig.1 Algorithm Flow Chart Overall

\subsection{Improved Surendra Algorithm}

In the original algorithm [4] [5], $\mathrm{T}$ is an empirical value and cannot be precisely determined. The variable $\mathrm{T}$ cannot adapt to the environment well when the circumstance

es is bad and has much lights and shades. Therefore, the factors that will affect the threshold value are studied in this paper.

In order to extract the moving target from the background, the extracted foreground is applied to find the pixel points $N_{1}$ of the moving target.

$$
N_{1}=\text { TotalPixels }-\left\|F_{i}\right\|_{1}
$$

Since the moving target is not at the same position between two frames, the target get longer after the difference processing. It means that the number of pixels $N_{2}$, which is processed by frame difference processing, will be nearly $\beta$ times as much ${ }_{\text {as }} N_{1}$.

The value of $\beta$ is specifically related to target speed, the interval time between two frames, and the distance between camera and target, but the speed of the vehicle distance changes little, and the distance between the camera and the road changes little. So $\beta$ is approximately related to the interval time between two 
frames. Thereby a research of the relationship between the interval time between the two frames and $\beta$ is carried on: Shown in Fig.2:

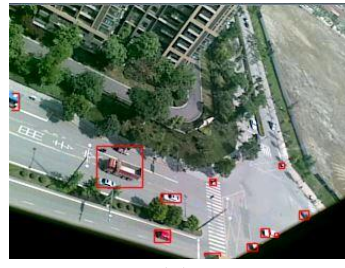

(a)

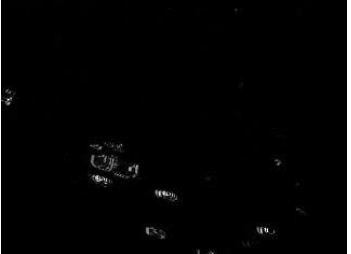

(b)

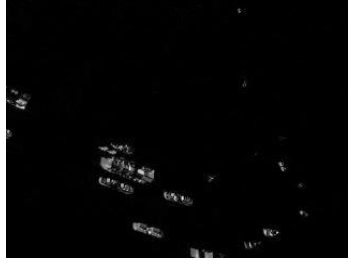

(c)

Fig.2 Compare between the differenced figure and the original figure. (a) is original figures; (b) is the differenced figure $I_{i}-I_{i-1}$; (c) is the differenced figure $I_{i}-I_{i-4}$. (Fps=15)

In figure 2 (b) and (c), the length of the vehicle is significantly longer than that in the original image. Therefore, increasing the number of test group could help figuring out the relationship between the interval time of the two frames which is Shown in the Fig.3:

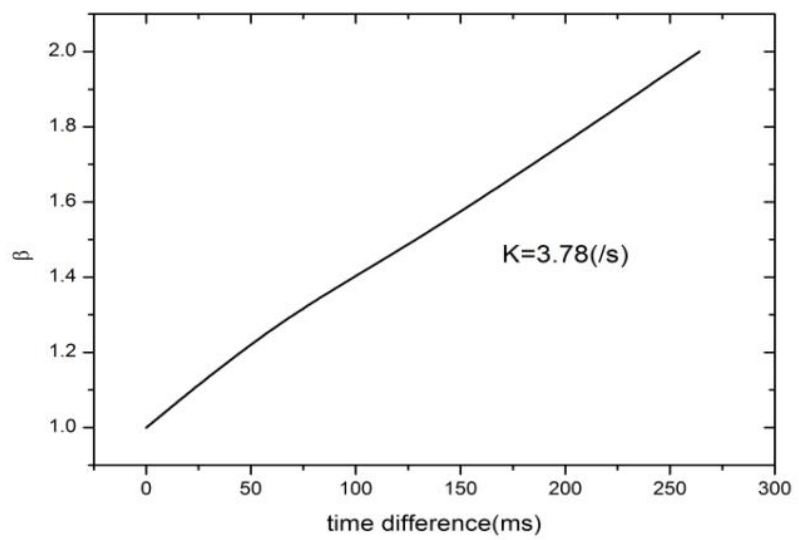

Fig.3 the fitting relation between $\beta$ and the intervals

We can conclude that $\beta$ and time interval are simple linear relationship. The relation is:

$$
\beta=1+3.78 \cdot N / f p s
$$

( $\mathrm{N}$ refers to the number of frames difference between two frames)

Considering that the information of the edge and the shadow of the cars in the separated foreground may be ignored, the interval time of the value of the $\beta$ needs to be expanded. Let:

$$
\beta=2+3.78 \cdot N / f p s
$$

Then calculate the number of each frame image pixels of background: 


$$
N_{B}=\text { TotalPixels }-N_{1} \cdot \beta
$$

Through the frame difference histogram, determine the threshold value in accordance with the below algorithm:

$$
\begin{gathered}
\text { thres }=i\left(\operatorname{when}\left(\operatorname{sum}\left(H_{i-1}\right)<N_{B} \wedge \operatorname{sum}\left(H_{i}\right)>N_{B}\right)\right) \\
\text { Here: } \operatorname{sum}\left(H_{i}\right) i s \sum_{0}^{i} H_{i}
\end{gathered}
$$

For each frame whose size is $240 \times 320$, time consumption of processing is just $8 \mathrm{~ms}$. This algorithm meets the need of real-time, even up to the frame-by-frame processing, at the same time also it has a high accuracy and good application prospect.

\subsection{The gradient update rate algorithm}

Taking the first frame as the initial background will lead to poor effect of extraction at the beginning of the video background, and thus there is no significance in the subsequent extraction. For this purpose we propose the gradient update rate method which is simple and effective. To be specifically, we find a suitable function to make the background update rate converge to default values at an appropriate speed. Because of the high update background rate, the initial background enter the " state" as soon as possible, and thus solving the problem of the moving target detection at the beginning of the video.

Gradient function used in this paper is an inverse proportional function:

$$
\alpha= \begin{cases}\frac{1}{n}+\alpha_{0} & n \leq 500 \\ \frac{2}{\alpha_{0}}+5 & n>500\end{cases}
$$

This function is simple in form, and the rate of decay tests appropriate. The new algorithm can make the background image of the video update quickly and stably at the beginning, and also its time-consuming is short, to sum up, which has certain practical value.

\section{Experiment results and analysis}

The experiment will set the first frame as background image, and adopting (and adopt) an improved algorithm of Surendra into real-time background updating. Hardware environment: 3G of memory; Intel(R) Core(TM) i3 CPU M 330 @ $2.13 \mathrm{GHz}$ 。Software environment: Windows 7; Microsoft Visual Studio 2010。Video frame rate of 15 frames / s, the frame size is $240 \times 320$. 


\subsection{Improved Surendra algorithm's performance}

Compare the improved Surendra algorithm with the commonly used OSTU algorithm, and the result is shown in Fig.4.

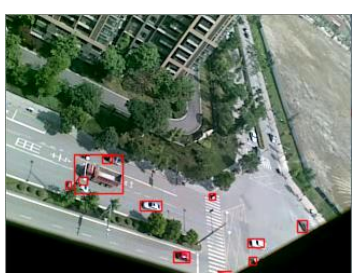

(a)

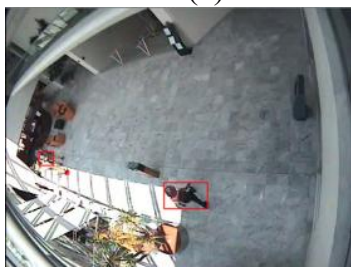

(d)

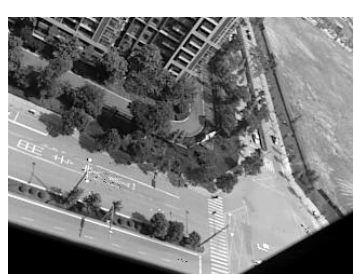

(b)

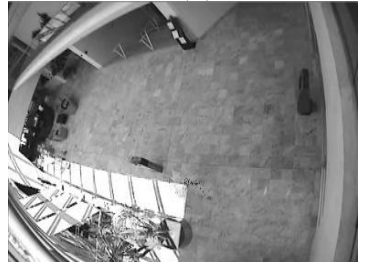

(e)

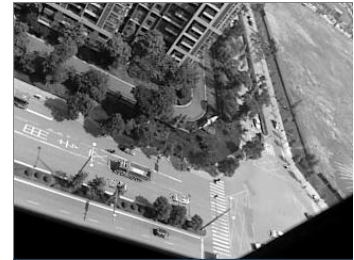

(c)

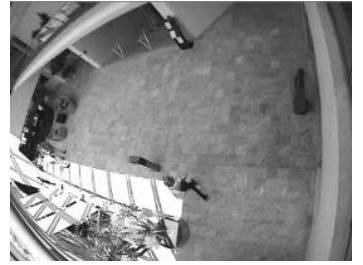

(f)

Fig.4 Compare between the improved surendra algorithm and OSTU algorithm from same original figure. (a) and (d) are the original figures;(b) and (e) are the results of the improved surendra algorithm from (a) and (d) respectively;(c) and (f) are the results of the OSTU algorithm from (a) and (d).

In the Fig.4 (b) (e), the moving vehicle has almost disappeared when the improved surendra algorithm proposed in this paper was used, and in the Fig.4(c)(f), the most parts of moving vehicle can still be seen when the OSTU algorithm was used; In Fig.4 (d) where there is lights and shades which is more complex to cope with, and it can be found that in the Fig. 4 (e) the person in the center of the figure has almost disappeared and in the Fig.4(f) the person can still be found. It shows the algorithm's superiority and robustness.

Table 1 Two algorithm's Time-Consuming:

\begin{tabular}{|l|c|}
\hline Algorithm & $\begin{array}{l}\text { Average Time } \\
\text { Consuming }\end{array}$ \\
\hline Improved Surendra Algorithm proposed in this paper & $0.008 \mathrm{~s}$ \\
\hline OSTU algorithm & $0.011 \mathrm{~s}$ \\
\hline
\end{tabular}

It shows that the new algorithm's time-consuming is smaller than the traditional OSTU algorithm, so it can save more time to the subsequent processing.

\subsection{The effect of gradient update rate algorithm (GUR algorithm)}

In order to know the effect of this new algorithm, using the 30th frame to find the difference shown in the Fig.5: 


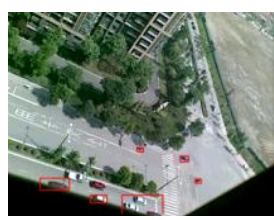

(a)

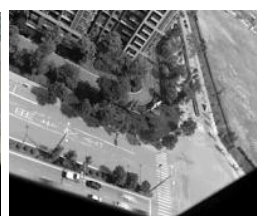

(b)

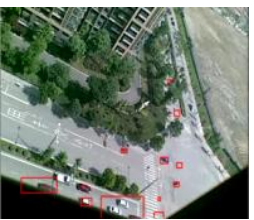

(c)

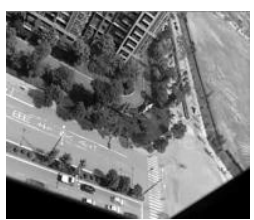

(d)

Fig.5 Two kinds of algorithm were used and the comparisons of two results were displayed above. (a) is the 30th foreground using the GUR algorithm;(b) is the 30th background using the GUR algorithm;(c) is the 30th foreground using original algorithm; (d) is the 30th background using the original algorithm.

It shows that using the gradient update rate algorithm proposed in this paper can effectively solve the shortcomings of the video background updating. In Fig.5 (c), there are a lot of background mistakes for a moving target, and this problem will no longer exist when the gradient update rate algorithm was used.

\subsection{Overall algorithm performance research}

In order to reflect the algorithm's real-time characteristics, using Gaussian mixture model algorithm and the average background algorithm comparing with the proposed algorithm is shown in table 1

Table 2 Several detection algorithms performance comparison

\begin{tabular}{|l|c|}
\hline Type of the Detection Algorithm & Time-Consuming Per Frame (s) \\
\hline Gaussian mixture model algorithm & 0.171 \\
\hline average background algorithm & 0.078 \\
\hline Algorithm proposed in this paper & 0.042 \\
\hline
\end{tabular}

From Table.1, Gaussian mixture model algorithm takes the longest time, and cannot do the frame-by-frame processing, and the algorithm in this paper is the fastest one to meet the need of real-time processing.

The overall algorithm experimental results have been shown in Fig.6. 


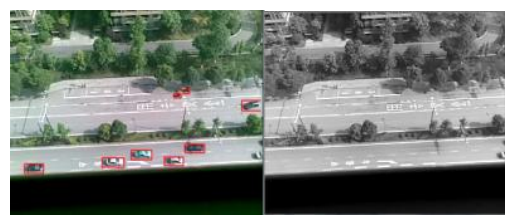

(a)

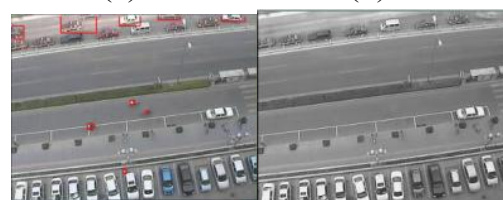

(e)

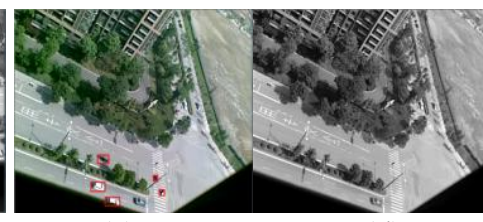

(c)

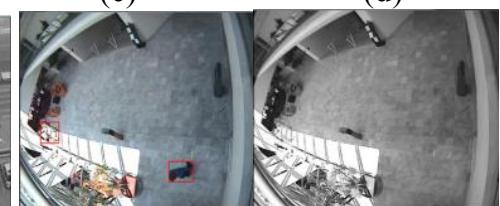

(g)

(h)

Fig.6 Compare between the detection result and the background. (a) (c) (e) (g) are the detection result from different video; (b) (d) (f) (h) are the background from different video correspondingly.

From Figure.6, moving targets is detected without omission no matter they are big or small. In Fig.6(c) (d), the blue car has stopped, the program regard them as background immediately. In Fig.6 (g) (h), the moving target can identified regardless of the brightness changes.

These fully proves the real-time and robustness of the improved algorithm, which thus can be applied in engineering applications.

\section{Conclusions}

This paper uses the improved Surendra algorithm to extract the background, combined with the gradient update rate algorithm to solve the problem of moving object detection in complex environments. The algorithm effectively solves the problem of the adaptive threshold selection of the Surendra algorithm, and give a good solution to the problem that the background image cannot be generated quickly at the beginning of the video .This algorithm has short time-consuming, high efficiency, strong anti-interference and adaptability to change in the external environment such as weather and light, and it can basically realize the real-time processing of moving object detection. The experiment reflects the proposed algorithm's effectiveness and robustness.

\section{Acknowledgments}

The author thanks Dr Lifeng Yang from University of electronic science and Technology of China in Chengdu for helpful guidance in how to do research on this field. This research was supported by a grant from the Innovation Fund offered by the School of Optoelectronic Information of the UESTC. 


\section{References}

1. Brown B, A Shafie A.A., Fadhlan Hafiz,Ali M.H., "Motion Detection Techniques Using Optical Flow," World Academy of Science, Engineering and Technology,559-561(2009).

2. Jialin Tang,Xiying Li, DongHua Luo, "Vehicle Detection at night Based on Frame Differenct," Computer Measurement \& Control,16(12),2008.

3. Zheng-qin Wang, FuQiang Liu, "Comparison of Adaptive Extract Algorithm of Object Scene," Computer Engineering, Vol.34(23),2008.

4. WANG Cheng-liang,JIA Liang-liang, CHEN Juan-Juan. Moving Object Detection and Tracking Based on Improved Surendra Background Updating Algorithm .Third Internationa Conference on Digital Image Processing[C] Vol. 8009(800919) 1-6(2013)5. South J, Blass B (2001) The future of modern genomics. Blackwell, London.

5. Feng Ding, Hong Wang, Hongsheng Zhong, Longhua Yu. Perimeter Intrusion Detection based on improved Surendra Background Update Algorithm .Third International Conference on Digital Image Processing [C] Vol. 8009(800938) 1-5(2013).

6. Li-na Qi,Bo Zhang, Zhan kai Wang, "Application of the OTSU Method in Image Processing," Radio Engineering,Vol.36(7),25-44(2006).

7. Lian Xiaofeng, Zhang Tao, Liu Zaiwen. A Novel Method on Moving-Objects Detection Based on Background Subtraction and Three Frames Differencing. 2010 International Conference on Measuring Technology and Mechatronics Automation, 10(132) 252-256(2010).

8. Yinq Zhu, Jiandeng Huang. Video background extraction based on improved mode algorithm[J]. $20093^{\text {rd }}$ International Conference on Genetic and Evolutionary Computing, 6(172)331-334(2009).

9. Huiling Zhang, Baichuan Lu, Yong Yu. The Method of Ostu Used in the Video Detection of Vehicles .International Conference on Intelligent Computation Technology and Automation[C] 547-551(2008). 